\title{
Plantar Pressure Distribution During Robotic- Assisted Gait in Post-stroke Hemiplegic Patients
}

\author{
Jin Kyu Yang, $\mathrm{MD}^{1,2}$, Na El Ahn², Dae Hyun Kim, MD ${ }^{1,2}$, Deog Young Kim, MD, PhD ${ }^{1,2}$ \\ ${ }^{1}$ Department of Rehabilitation Medicine and ${ }^{2}$ Research Institute of Rehabilitation Medicine, \\ Yonsei University College of Medicine, Seoul, Korea
}

Objective To assess the plantar pressure distribution during the robotic-assisted walking, guided through normal symmetrical hip and knee physiological kinematic trajectories, with unassisted walking in post-stroke hemiplegic patients.

Methods Fifteen hemiplegic stroke patients, who were able to walk a minimum of ten meters independently but with asymmetric gait patterns, were enrolled in this study. All the patients performed both the robotic-assisted walking (Lokomat) and the unassisted walking on the treadmill with the same body support in random order. The contact area, contact pressure, trajectory length of center of pressure (COP), temporal data on both limbs and asymmetric index of both limbs were obtained during both walking conditions, using the F-Scan in-shoe pressure measurement system.

Results The contact area of midfoot and total foot on the affected side were significantly increased in roboticassisted walking as compared to unassisted walking $(\mathrm{p}<0.01)$. The contact pressure of midfoot and total foot on affected limbs were also significantly increased in robotic-assisted walking $(\mathrm{p}<0.05)$. The anteroposterior and mediolateral trajectory length of COP were not significantly different between the two walking conditions, but their trajectory variability of COP was significantly improved $(\mathrm{p}<0.05)$. The asymmetric index of area, stance time, and swing time during robotic-assisted walking were statistically improved as compared with unassisted walking $(\mathrm{p}<0.05)$.

Conclusion The robotic-assisted walking may be helpful in improving the gait stability and symmetry, but not the physiologic ankle rocker function.

Keywords Robotics, Walking, Hemiplegia, Plantar pressure, Gait symmetry

Received August 8, 2013; Accepted October 15, 2013

Corresponding author: Deog Young Kim

Department of Rehabilitation Medicine and Research Institute of Rehabilitation Medicine, Yonsei University College of Medicine, 50 Yonsei-ro, Seodaemun-gu, Seoul 120-749, Korea

Tel: +82-2-2228-3714, Fax: +82-2-363-2795, E-mail: KIMDY@yuhs.ac

(c) This is an open-access article distributed under the terms of the Creative Commons Attribution Non-Commercial License (http://creativecommons. org/licenses/by-nc/3.0) which permits unrestricted noncommercial use, distribution, and reproduction in any medium, provided the original work is properly cited.

Copyright $\odot 2014$ by Korean Academy of Rehabilitation Medicine

\section{INTRODUCTION}

Stroke is a leading cause of neurological impairment in adults [1]. Impaired walking function, one of the most devastating disabilities in post-stroke hemiplegic patients, greatly contributes to functional disability and safety following the onset of stroke [2]. The restoration of walking is the goal most often stated by post-stroke patients and identified as one of the most important rehabilitation goals in the stroke patients [2]. The gait training 
has been largely based on physical therapy interventions including the Bobath technique, task-oriented training techniques and bodyweight support treadmill training [3]. However, conventional gait training does not restore the normal gait pattern in the majority of stroke patients [4].

Recently, robotic-assisted locomotor gait techniques have been introduced with emerging approaches to gait rehabilitation of individuals after stroke, and they are being increasingly accepted among researchers and clinicians [5]. These robotic devices provide a safe, intensive and task-oriented rehabilitation for people with mildto-severe motor impairments following a stroke [6]. The recent Cochrane reviews have revealed that patients who received robotic-assisted gait training in combination with physiotherapy after stroke were more likely to achieve independent walking than patients receiving gait training without such devices [7].

These robotic gait trainers are designed to guide a subject through a normal physiological gait pattern with precisely controllable assistance during movement training [8]. Among these, Lokomat (Hocoma AG, Volketswil, Switzerland) is the most popular robotic-assisted gait trainer. It is composed of a treadmill, bodyweight support system and two light-weight robotic actuators with a fully programmable control of knee and hip range of motion [9]. However, the ankle control is not provided by programmable robotic actuators, and only instrumented footlifters are applied to feet to aid with foot clearance during swing [8]. Hidler and Neckel [9] showed that the hip, knee, and ankle moments during gait training with Lokomat (Hocoma AG) were similar to those reported during overground gait in healthy persons using a 3-D inverse dynamics-based approach. Neckel et al. [8] reported, however, that post-stroke subjects produced similar overall kinematic patterns on the sagittal plane, except for decreased ankle ROM during robotic-assisted gait training. They also generated abnormal joint torque patterns, particularly in the affected limb.

The ankle and foot are the main structures that support the body, and their abnormal motions affect the balance of legs and trunk and cause the change of overall gait pattern [10]. It is unclear whether robotic-assisted gait trainers guided through normal symmetrical hip and knee physiological kinematic trajectories also result in symmetric, physiological functions of ankle, which is not driven by the robotic-assisted gait trainer.

The sense of balance and movement pattern of subjects could be evaluated by observing the transient moving path of center of pressure (COP) during the gait cycle [11]. The measurement of COP is done using plantar pressure measurement system, considered to be a reliable and objective test [12].

We conducted this study to investigate the ankle function and gait asymmetry during the robotic-assisted walking, which induces the normal physiological hip and knee motion. We compared the robotic-assisted walking with the unassisted walking on the same bodyweight supported treadmill in hemiplegic stroke patients.

\section{MATERIALS AND METHODS}

\section{Subjects}

Fifteen hemiplegic stroke patients (10 men and 5 women; interval from stroke 7.9 months; range 1.4-46.0 months; hemisphere of stroke, 6 right and 9 left) with mean age of 50.7 years were enrolled in our study. The types of stroke were infarction in eleven and hemorrhage in four patients. Mean lower extremity score of the Fugl-Meyer Motor Assessment Scale [13] was 25.6 (range 17-33); and Functional Ambulation Category [14] was 3.2 (range 3-4). All the subjects' ankle motion was passively full, and fixed deformities were not seen. The manual muscle test of ankle dorsiflexor ranged from $P$ grade to G grade (good, 2; fair+, 1; fair, 3; fair-, 3; poor+, 1; poor, 2 ; poor-, 3 persons). All patients walked independently without ankle foot orthosis. The foot drop was observed in twelve subjects, and the ankle inversion was observed in seven patients during walking. In all patients, diagnosis of stroke was established by magnetic resonance imaging. Patients who met the following eligibility criteria were included: 1) first-ever unilateral stroke (hemorrhagic or ischemic); 2) 18 years or older; 3) normal gait pattern before first-ever stroke; and 4) Functional Ambulation Category [14] score of 3 or more. Patients were excluded if they had a presence of subarachnoid hemorrhage, sequelae of previous neurologic or orthopedic disorder that could impair locomotion, limited range of motion or severe spasticity of lower extremity, skin problems, severe cognitive or visuospatial dysfunction and/or severe medical illness. All the patients were informed of the study and submitted a written informed consent before 
enrollment.

\section{Study design}

All patients performed both the robotic-assisted walking and unassisted walking on the same day in a random order. In robotic-assisted walking, robotic-assisted gait orthosis was applied to both legs. The orthosis was not applied in unassisted walking. Lokomat (Hocoma AG) was run with $100 \%$ guidance force under trial, with the device in pure position control mode rather than impedance mode in robotic-assisted walking. Both trials were performed under the same conditions with $50 \%$ bodyweight support in the same patient, who selected the walking speed of the treadmill. To familiarize with the trials, patients tried the robotic-assisted and unassisted walking in at least three 30 -minute sessions, until they felt comfortable on the day before the trials.

\section{Measurements}

Measurements were recorded at $50 \mathrm{~Hz}$ by F-Scan system (Tekscan Inc., Boston, MA, USA). As according to the Tekscan user manual (Tekscan Research Software User Manual version 6.7 Rev. D, 2003), we calibrated to zero the condition of supporting $50 \%$ bodyweight without foot-contact on the treadmill once the foot-straps were applied. Such minimized the foot-strap induced errors during the robotic-assisted walking. The calibration was done in the same manner for unassisted walking on the treadmill, excepting the foot-strap. The plantar pressures were measured using pressure insoles, which had 960 force-sensing resistors (3.88 sensors per centimeter square) and were $0.16-\mathrm{mm}$ thick. The insoles, trimmed to each subject foot size, were put in each shoe for both walking conditions, after removing the subject's own insoles.

All data was obtained during a 10-gait cycle of 10-minute walking trial to minimize the effects of fluctuating bodyweight support, as shown at initial and terminal waking periods.

The time of swing phase and stance phase was measured. Stance phase was sub-classified into the initial double-limb support, single-limb support, and terminal double-limb support. The contact area was measured as the sum of area that contacted the floor of treadmill floor during one gait cycle. The contact pressure was measured as a sum of the mean pressures, measured at each pres-

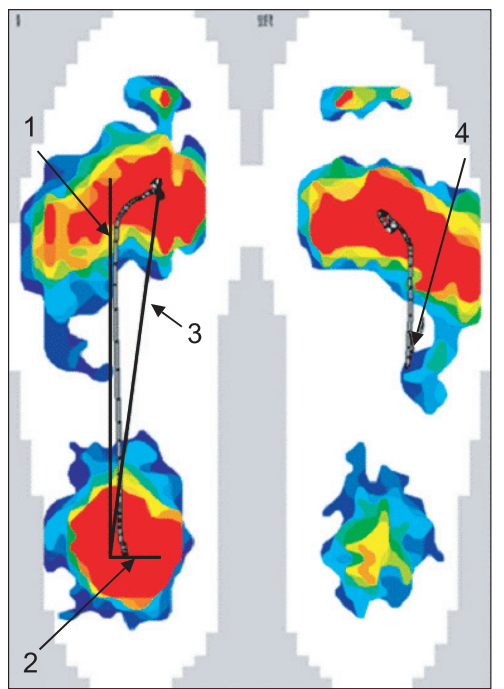

Fig. 1. Trajectory length and back movement number using path of the center of pressure were measured based on the study of Paik and Im [11]. 1, Anterior-posterior trajectory; 2, mediolateral trajectory; 3 , total trajectory; 4 , back movement number.

sure sensor during one gait cycle. Both the contact area and pressure were divided into those of anterior onethird (forefoot), middle one-third (midfoot) and posterior one-third (hindfoot), based on each subject's anteroposterior foot length. The trajectory length and frequency of back movement were measured using the path of COP, as based on the reports of Paik and Im [11] (Fig. 1). The anteroposterior and mediolateral trajectory variability were measured as the standard deviation of anteroposterior and mediolateral trajectory length of 10-gait cycles.

The patterns of vertical ground reaction force were defined as: 1, irregular shape; 2, inverted V pattern; 3, inverted U pattern; and 4, bimodal M pattern [15]. The asymmetry of gait data, including temporal data, contact area, contact pressure, and trajectory length, were calculated according to the following formula [16]:

$$
\left[\left(\mathrm{V}_{\text {paretic }}-\mathrm{V}_{\text {non-paretic }}\right) / 0.5\left(\mathrm{~V}_{\text {paretic }}+\mathrm{V}_{\text {non-paretic }}\right)\right] \times 100(\%)
$$

\section{Statistical analysis}

Statistical analysis was performed using SPSS ver. 20.0 (IBM, Armonk, NY, USA). All measurements, except the pattern of the vertical ground reaction force, were analyzed for the difference between the robotic-assisted walking and unassisted walking using Wilcoxon signedrank test. The pattern of ground reaction force was com- 
pared using the Bowker symmetry test. The difference was considered to significant at $\mathrm{p}<0.05$.

\section{RESULTS}

\section{Temporal data}

The single-limb support time and the terminal doublelimb support time on the affected side were significantly increased in robotic-assisted walking as compared to unassisted walking $(\mathrm{p}<0.01)$. Other temporal parameters did not show a statistically significant difference. The initial double-limb support time and the swing time on unaffected limb were significantly increased in robotic-assisted walking as compared to unassisted walking $(\mathrm{p}<0.01)$. Other time parameters did not show a statistically significant difference. Additional data is presented in Table 1.

\section{Contact area and pressure}

The contact area of midfoot and total foot on the af- fected side were significantly increased in roboticassisted walking $(\mathrm{p}<0.01)$. However, the contact area of forefoot and hindfoot on affected side did not show statistically significant difference. Also, the contact pressure of midfoot and total contact pressure of affected limbs were also significantly increased in robotic-assisted walking, as compared with unassisted walking $(\mathrm{p}<0.01)$. Those of unaffected midfoot also significantly increased in robotic-assisted walking $(\mathrm{p}<0.05)$. Other contact area and pressures did not show statistical significance. The peak pressure on the unaffected side were significantly increased in robotic-assisted walking than in unassisted walking $(\mathrm{p}<0.01)$, but that of affected side were not. The details of contact area and pressure and peak pressure during both conditions of walking are shown in Table 2 .

\section{Trajectory}

The anteroposterior, mediolateral, and total trajectory length did not show statistically significant differences

Table 1. Comparison of temporal data between robotic-assisted walking and unassisted walking (unit, second)

\begin{tabular}{lccccc}
\hline \multirow{2}{*}{ Parameter } & \multicolumn{2}{c}{ Affected } & & \multicolumn{2}{c}{ Unaffected } \\
\cline { 2 - 3 } \cline { 5 - 6 } & Robotic-assisted & Unassisted & & Robotic-assisted & Unassisted \\
\hline Stance phase & $2.30 \pm 0.89$ & $1.21 \pm 0.36$ & & $2.06 \pm 0.54$ & $1.35 \pm 0.42$ \\
Initial double-limb support & $0.48 \pm 0.34$ & $0.34 \pm 0.30$ & & $0.67 \pm 0.48^{* *}$ & $0.23 \pm 0.12$ \\
Single-limb support & $1.16 \pm 0.37^{* *}$ & $0.62 \pm 0.16$ & & $0.92 \pm 0.51$ & $0.77 \pm 0.28$ \\
Terminal double-limb support & $0.64 \pm 0.48^{* *}$ & $0.24 \pm 0.13$ & & $0.47 \pm 0.35$ & $0.35 \pm 0.30$ \\
\hline Swing phase & $0.94 \pm 0.51$ & $0.77 \pm 0.26$ & & $1.16 \pm 0.37^{* *}$ & $0.62 \pm 0.17$ \\
\hline
\end{tabular}

Values are presented as mean \pm standard deviation.

${ }^{*} \mathrm{p}<0.05,{ }^{* *} \mathrm{p}<0.01$.

Table 2. Comparison of contact area and pressure between robotic-assisted walking and unassisted walking

\begin{tabular}{llccccc}
\hline \multirow{2}{*}{ Parameter } & \multirow{2}{*}{ Site } & \multicolumn{2}{c}{ Affected } & & \multicolumn{2}{c}{ Unaffected } \\
\cline { 6 - 7 } \cline { 5 - 6 } & & Robotic-assisted & Unassisted & & Robotic-assisted & Unassisted \\
\hline Contact area $\left(\mathrm{cm}^{2}\right)$ & Forefoot & $26.34 \pm 15.17$ & $23.78 \pm 11.87$ & & $29.27 \pm 11.52$ & $28.32 \pm 13.49$ \\
& Midfoot & $48.15 \pm 16.88^{* *}$ & $35.37 \pm 15.60$ & & $44.35 \pm 14.56$ & $41.25 \pm 15.94$ \\
& Hindfoot & $28.35 \pm 11.57$ & $20.78 \pm 12.79$ & & $29.73 \pm 9.24$ & $30.04 \pm 9.25$ \\
& Total & $102.81 \pm 36.77^{* *}$ & $79.93 \pm 32.50$ & & $103.35 \pm 23.33$ & $99.62 \pm 32.03$ \\
\hline Contact pressure $\left(10^{3} \mathrm{kPa}\right)$ & Forefoot & $49.26 \pm 48.97$ & $50.20 \pm 48.22$ & & $86.92 \pm 69.98$ & $79.14 \pm 67.74$ \\
& Midfoot & $98.06 \pm 58.90^{* *}$ & $63.08 \pm 29.53$ & & $93.73 \pm 38.65^{*}$ & $75.72 \pm 33.10$ \\
\hline & Hindfoot & $62.83 \pm 46.60$ & $34.56 \pm 25.87$ & & $90.71 \pm 91.14$ & $73.65 \pm 64.28$ \\
\hline Peak pressure $\left(10^{3} \mathrm{kPa}\right)$ & Total & $210.15 \pm 133.05^{*}$ & $147.84 \pm 77.27$ & & $271.35 \pm 135.02^{* *}$ & $228.51 \pm 125.77$ \\
\hline
\end{tabular}

Values are presented as mean \pm standard deviation.

${ }^{*} \mathrm{p}<0.05,{ }^{* *} \mathrm{p}<0.01$. 
Table 3. Comparison of trajectory length and variability, number of back movement and vertical ground reaction force pattern between robotic-assisted walking and unassisted walking (unit, $\mathrm{cm}$ )

\begin{tabular}{lccccc}
\hline \multirow{2}{*}{ Parameter } & \multicolumn{2}{c}{ Affected } & & \multicolumn{2}{c}{ Unaffected } \\
\cline { 2 - 3 } \cline { 5 - 6 } & Robotic-assisted & Unassisted & & Robotic-assisted & Unassisted \\
\hline Trajectory length & $8.48 \pm 3.01$ & $6.92 \pm 3.33$ & & $11.21 \pm 2.99$ & $12.26 \pm 3.74$ \\
\hline Anteroposterior & $1.72 \pm 0.44$ & $1.78 \pm 0.54$ & & $1.89 \pm 0.44$ & $2.00 \pm 0.70$ \\
\hline Mediolateral & $8.56 \pm 2.96$ & $7.13 \pm 3.24$ & & $11.33 \pm 2.99$ & $12.39 \pm 3.76$ \\
\hline Total & & & & $0.84 \pm 0.88$ & $1.01 \pm 0.84$ \\
\hline Variability & $0.31 \pm 0.14^{* *}$ & $0.96 \pm 0.36$ & & $0.22 \pm 0.10$ & $0.29 \pm 0.19$ \\
\hline Anteroposterior & $0.15 \pm 0.08^{*}$ & $0.29 \pm 0.16$ & & $3.51 \pm 2.38^{*}$ & $2.17 \pm 1.08$ \\
\hline Mediolateral & $3.59 \pm 1.66$ & $3.47 \pm 1.64$ & & $1.89 \pm 0.44$ & $2.00 \pm 0.70$ \\
\hline Number of back movement & $9: 5: 1: 0$ & $12: 2: 1: 0$ & & $8: 3: 4: 0$ & $4: 8: 2: 1$ \\
\hline GRFP (1:2:3:4) & & & &
\end{tabular}

Values are presented as mean \pm standard deviation.

GRFP, ground reaction force pattern.

${ }^{*} \mathrm{p}<0.05,{ }^{* *} \mathrm{p}<0.01$.

Table 4. Asymmetric index of robotic-assisted walking condition and unassisted walking condition

\begin{tabular}{lcc}
\hline \multicolumn{1}{c}{ Parameter } & Robotic assisted & Unassisted \\
\hline Stance time & $0.13 \pm 0.50^{*}$ & $-0.57 \pm 1.45$ \\
Swing time & $-0.57 \pm 1.45^{*}$ & $0.40 \pm 0.83$ \\
Contact area & $-5.84 \pm 27.74^{*}$ & $-25.21 \pm 20.35$ \\
Contact pressure & $-36.54 \pm 66.52$ & $-43.27 \pm 46.59$ \\
AP trajectory & $-30.29 \pm 41.40$ & $-58.98 \pm 52.78$ \\
ML trajectory & $-8.78 \pm 41.15$ & $-8.51 \pm 51.55$ \\
Total trajectory & $-30.48 \pm 40.94$ & $-55.79 \pm 49.25$ \\
\hline
\end{tabular}

Values are presented as mean \pm standard deviation.

AP, anteroposterior; ML, mediolateral.

${ }^{*} \mathrm{p}<0.05$.

between the two walking conditions regardless of the limb side. However, the variability of anteroposterior and mediolateral trajectory on the affected side was significantly improved in robotic-assisted walking as compared to unassisted walking $(\mathrm{p}<0.05)$. The pattern of the vertical ground reaction force also did not show a statistically significant difference between the two walking conditions. The frequency of back movement of the unaffected side showed a statistically significant increase in roboticassisted walking as compared to unassisted walking $(\mathrm{p}<0.05)$. However, the frequency of back movement on the affected side did not show a statistically significant difference. Additional data is presented in Table 3.

\section{Gait asymmetry}

In robotic-assisted walking, the asymmetric index of contact area, stance time, and swing time between the two lower limbs were significantly improved as compared with unassisted walking $(\mathrm{p}<0.05)$. Those of contact pressure and trajectory length did not show a statistically significant difference (Table 4).

\section{DISCUSSION}

This is the first study that investigated whether roboticassisted gait trainers, guided through normal symmetrical hip and knee physiological kinematic trajectories, resulted in symmetric, physiological functions of ankle. The results of this study indicated that robotic-assisted gait training may improve the gait symmetry and stability, but the ankle rocker function did not.

In overground walking, the contact pressure and area of the affected side in hemiplegic patients with stroke are markedly decreased as compared with those of the unaffected side. Hence, the asymmetry of the contact pressure and area was observed [17]. The abnormal muscle activity, abnormal posture and disturbance of sense made patients with hemiplegia load their weight distribution to the unaffected legs [18-20]. The asymmetrical weight-bearing of the patients continued during their walking as well as standing, which resulted in asymmetric and inefficient gait and increased risk of falling [20,21]. In this regard, Brunt et al. [22] reported on the correla- 
tion between weight-bearing capacity and the power to initiate walk to emphasize the treatment for symmetrical weight-bearing. Similarly, symmetrical walking after stroke was an important indicator for restoration [23]. In our study, the asymmetry of contact area, with increased contact area and pressures of midfoot and total foot, was improved during robotic-assisted walking as compared with unassisted walking. These findings indicate that robotic-assisted walking may improve the gait though symmetric weight distribution of the robotic-assisted gait trainer. There is improved stability on the affected side during walking through the firm instrumented shank and thigh cuffs. The effects of foot-strap can fix the dynamic equinovarus deformity during walking. It may be helpful for providing sufficient sensory stimulation on post-stoke hemiplegic patients' feet.

Moreover, the asymmetric pattern of stance time and swing time were markedly improved during roboticassisted walking gait in this study. These findings support that the robotic-assisted walking gait can help the poststroke hemiplegic victims to have more symmetric gait patterns. Miyai et al. [24] reported that the locomotor recovery after stroke was associated with improvement of asymmetry in primary sensorimotor cortex activation and enhancement of premotor cortex activation in the affected hemisphere. Therefore, robotic-assisted walking gait training may be considered to have a positive influence on neuroplasty after a stroke. However, the midfoot pressure of the unaffected side was also elevated. It was expected that the foot-straps contacted and pressured the subjects' feet regardless of the involved side, despite the zero-calibration to minimize the effects of foot-strap pressure. However, such may not be considered as the main effect for the change of feet contact area and pressure, as the asymmetry of contact area was also improved and the foot-strap had the same pressure on both feet regardless of the involved side.

Many researchers have reported that the stance time and single-limb supporting time are shorter in poststroke hemiplegic patients as compared with normal healthy persons $[5,7,8,16]$. In this study, the stance time on the affected side (especially, the single-limb supporting time) was significantly increased. The swing time on the unaffected side was also significantly increased during robotic-assisted walking. These findings are in agreement with previous reports that the robotic-assisted walking gait increased the duration of single-limb support on the paretic leg [25]. We thought that the roboticassisted gait orthosis may produce stability on the singlelimb supporting time for the affected side.

Human walking appears to be passively unstable, requiring active feedback control for stability [26]. The stability during walking is more disturbed in post-stroke hemiplegic patients [17]. In this study, the variability of anteroposterior and mediolateal trajectory on the affected side was significantly decreased during roboticassisted walking as compared to the control walking conditions. Increased variability was shown in hemiplegic patients. Such findings indicate that robotic-assisted walking gait may help the affected leg to be more stable in walking. It is consistent with the reports of Donelan et al. [26], where the external lateral stabilization using springs that pulled bilaterally from the waist resulted in a decrease of the variability in the step-width and a decrease in the metabolic cost.

Typically, the trajectory length is shorter and the frequency of back movement higher on the affected side during walking in post-stroke patients [27]. Previous studies have reported not only that the Lokomat robotic orthosis guided a subject through a symmetric physiological gait pattern as they walked on a treadmill with or without bodyweight support $[5,28]$, but that the vertical ground reaction force during robotic-assisted walking was also similar to that of normal healthy persons [8]. However, our results did not support the opinion that the robotic-assisted walking could induce the physiologic ankle rocker function. In this study, the anteroposterior trajectory length and the frequency of back movement on the affected side during robotic-assisted walking were not improved as compared with unassisted walking. In addition, the pattern of the vertical ground reaction force also showed no statistically significant difference between robotic-assisted walking and unassisted conditions. These findings are consistent with the reports of Neckel et al. [8], where the ankle range of motion on the impaired limb was decreased. The peak ankle plantarflexion at push-off was particularly decreased during robotic-assisted walking. Since the Lokomat does not drive the ankle directly, passive foot-straps were applied to the impaired ankle. As such, assisting ankle dorsiflexion may limit the extent of ankle plantarflexion. It can therefore be inferred that the insufficient third ankle rocker could not make the tra- 
jectory longer during robotic-assisted walking. Another possible reason can be found for the reports of Gottschall and Kram [29] that the bodyweight support during walking may alter the muscular work for propulsion. They reported that the bodyweight supporting during walking decreased the mean EMG of the medial gastrocnemius during the propulsive phase. Moreover, our findings are consistent with previous reports that lower extremity muscle activity $[30,31]$, acceleration patterns of the lower limbs [32] and joint movements [8,9] produced by participants are altered in robotic-assisted walking, as compared with normal human walking. Based on our findings, it can be speculated that robotic-assisted walking cannot fix stereotypical abnormal motor behaviors in the lower extremities of post-stroke hemiplegic patients. As reported by Perry [33], maintaining the asymmetrical walking pattern as a compensation for patients whose forward movement cannot completely be controlled may have negative effect in improving a more physiological gait pattern in post-stroke hemiplegic patients.

This study has limiting factors. As the patients showed variable, abnormal gait patterns, we could not assess whether our findings were influenced by gait patterns. Another is the effect of foot-strap on our findings during the robotic-assisted walking. The unavoidable footstrap may influence the plantar pressure during roboticassisted walking. We calibrated to minimize the effects of foot-straps, but we could not completely exclude their possible effects.

In conclusion, our findings suggest that robotic-assisted gait orthosis (Lokomat) may improve the gait stability and symmetry, but not the ankle rocker function. It may be helpful to predict and understand the outcomes after robotic-assisted gait training in clinical settings. Further studies are warranted to examine whether long-term training with devices such as the Lokomat are effective in improving the gait patterns and walking ability in hemiparetic stroke subjects.

\section{CONFLICT OF INTEREST}

No potential conflict of interest relevant to this article was reported.

\section{ACKNOWLEDGMENTS}

This work was supported by the Professor Research
Funds of Yonsei University College of Medicine in 2012 (6-2012-0024).

\section{REFERENCES}

1. Whitall J. Stroke rehabilitation research: time to answer more specific questions? Neurorehabil Neural Repair 2004;18:3-8.

2. Bohannon RW, Horton MG, Wikholm JB. Importance of four variables of walking to patients with stroke. Int J Rehabil Res 1991;14:246-50.

3. Belda-Lois JM, Mena-del Horno S, Bermejo-Bosch I, Moreno JC, Pons JL, Farina D, et al. Rehabilitation of gait after stroke: a review towards a top-down approach. J Neuroeng Rehabil 2011;8:66.

4. Dohring ME, Daly JJ. Automatic synchronization of functional electrical stimulation and robotic assisted treadmill training. IEEE Trans Neural Syst Rehabil Eng 2008;16:310-3.

5. Hidler J, Nichols D, Pelliccio M, Brady K. Advances in the understanding and treatment of stroke impairment using robotic devices. Top Stroke Rehabil 2005;12:22-35.

6. Fasoli SE, Krebs HI, Stein J, Frontera WR, Hughes R, Hogan N. Robotic therapy for chronic motor impairments after stroke: follow-up results. Arch Phys Med Rehabil 2004;85:1106-11.

7. Mehrholz J, Werner C, Kugler J, Pohl M. Electromechanical-assisted training for walking after stroke. Cochrane Database Syst Rev 2007;(4):CD006185.

8. Neckel ND, Blonien N, Nichols D, Hidler J. Abnormal joint torque patterns exhibited by chronic stroke subjects while walking with a prescribed physiological gait pattern. J Neuroeng Rehabil 2008;5:19.

9. Hidler J, Neckel N. Inverse-dynamics based assessment of gait using a robotic orthosis. Conf Proc IEEE Eng Med Biol Soc 2006;1:185-8.

10. Park ES, Park CI, Kim JY, Park JW, Kim EJ. Foot pressure distribution and path of center of pressure (COP) of foot during ambulation in the children with spastic cerebral palsy. J Korean Acad Rehabil Med 2002;26:127-32.

11. Paik NJ, Im MS. The path of center of pressure (COP) of the foot during walking. J Korean Acad Rehabil Med 1997;21:762-71.

12. Ahroni JH, Boyko EJ, Forsberg R. Reliability of F-scan in-shoe measurements of plantar pressure. Foot Ankle 
Int 1998;19:668-73.

13. Malouin F, Pichard L, Bonneau C, Durand A, Corriveau $\mathrm{D}$. Evaluating motor recovery early after stroke: comparison of the Fugl-Meyer Assessment and the Motor Assessment Scale. Arch Phys Med Rehabil 1994;75:1206-12.

14. Holden MK, Gill KM, Magliozzi MR, Nathan J, PiehlBaker L. Clinical gait assessment in the neurologically impaired: reliability and meaningfulness. Phys Ther 1984;64:35-40.

15. Chen CY, Hong PW, Chen CL, Chou SW, Wu CY, Cheng PT, et al. Ground reaction force patterns in stroke patients with various degrees of motor recovery determined by plantar dynamic analysis. Chang Gung Med J 2007;30:62-72.

16. Patterson KK, Gage WH, Brooks D, Black SE, McIlroy WE. Evaluation of gait symmetry after stroke: a comparison of current methods and recommendations for standardization. Gait Posture 2010;31:241-6.

17. Kim DY, Park CI, Jang YW, Ahn SY, Na SI, Park YS. The relationship between weight-bearing and stiff-knee gait in hemiplegic patients. J Korean Acad Rehabil Med 2004;28:20-5.

18. Arcan M, Brull MA, Najenson T, Solzi P. FGP assessment of postural disorders during the process of rehabilitation. Scand J Rehabil Med 1977;9:165-8.

19. Wall JC, Ashburn A. Assessment of gait disability in hemiplegics: hemiplegic gait. Scand J Rehabil Med 1979;11:95-103.

20. Wall JC, Turnbull GI. Gait asymmetries in residual hemiplegia. Arch Phys Med Rehabil 1986;67:550-3.

21. Chaudhuri S, Aruin AS. The effect of shoe lifts on static and dynamic postural control in individuals with hemiparesis. Arch Phys Med Rehabil 2000;81:1498503.

22. Brunt D, Vander Linden DW, Behrman AL. The relation between limb loading and control parameters of gait initiation in persons with stroke. Arch Phys Med Rehabil 1995;76:627-34.
23. Roerdink M, Beek PJ. Understanding inconsistent step-length asymmetries across hemiplegic stroke patients: impairments and compensatory gait. Neurorehabil Neural Repair 2011;25:253-8.

24. Miyai I, Yagura H, Hatakenaka M, Oda I, Konishi I, Kubota K. Longitudinal optical imaging study for locomotor recovery after stroke. Stroke 2003;34:2866-70.

25. Hidler J, Nichols D, Pelliccio M, Brady K, Campbell $\mathrm{DD}$, Kahn JH, et al. Multicenter randomized clinical trial evaluating the effectiveness of the Lokomat in subacute stroke. Neurorehabil Neural Repair 2009;23:5-13.

26. Donelan JM, Shipman DW, Kram R, Kuo AD. Mechanical and metabolic requirements for active lateral stabilization in human walking. J Biomech 2004;37:82735.

27. Chisholm AE, Perry SD, McIlroy WE. Inter-limb centre of pressure symmetry during gait among stroke survivors. Gait Posture 2011;33:238-43.

28. Colombo G, Joerg M, Schreier R, Dietz V. Treadmill training of paraplegic patients using a robotic orthosis. J Rehabil Res Dev 2000;37:693-700.

29. Gottschall JS, Kram R. Energy cost and muscular activity required for propulsion during walking. J Appl Physiol (1985) 2003;94:1766-72.

30. Israel JF, Campbell DD, Kahn JH, Hornby TG. Metabolic costs and muscle activity patterns during robotic- and therapist-assisted treadmill walking in individuals with incomplete spinal cord injury. Phys Ther 2006;86:1466-78.

31. Hidler JM, Wall AE. Alterations in muscle activation patterns during robotic-assisted walking. Clin Biomech (Bristol, Avon) 2005;20:184-93.

32. Regnaux JP, Saremi K, Marehbian J, Bussel B, Dobkin $\mathrm{BH}$. An accelerometry-based comparison of 2 robotic assistive devices for treadmill training of gait. Neurorehabil Neural Repair 2008;22:348-54.

33. Perry J. The mechanics of walking in hemiplegia. Clin Orthop Relat Res 1969;63:23-31. 